\title{
Faricimab: expanding horizon beyond VEGF
}

\author{
Ashish Sharma $^{1}$ - Nilesh Kumar $\mathbb{D}^{1} \cdot$ Baruch D. Kuppermann ${ }^{2} \cdot$ Francesco Bandello $^{3}$ - Anat Loewenstein ${ }^{4}$
}

Received: 31 July 2019 / Accepted: 26 September 2019 / Published online: 6 November 2019

(c) The Royal College of Ophthalmologists 2019

With the advent of anti-vascular endothelial growth factor (anti-VEGF) therapy, there has been a significant reduction in disease burden attributable to retinal vascular pathologies. These molecules have been limited by their focused action on VEGF, and thus have phenomena such as resistance, non-response and recurrences of pathology in many cases. This led the researchers to look beyond anti-VEGF molecules. The recent research indicates that there are factors other than VEGF involved in pathogenesis. One of the most prominent among these factors is angiopoietin (Ang), which works via Ang-Tie receptor axis [1].

Angiopoietins bind to tyrosine kinase (Tie-2) endothelial receptors and regulate vasculogenesis. Ang-1 and Ang-2 are two isoforms and bind to Tie-2 with similar efficacy. While Ang-1 is a strong agonist of the Tie-2 receptor, Ang-2 acts as an antagonist inhibiting tie-2 phosphorylation [2, 3]. Evidences suggest upregulation of Ang-2 destabilises the endothelial cell layer leading to fluid leakage. It also makes the endothelial cells more responsive to VEGF and other proangiogenic mediators [4].

Bispecific antibodies can help target two of these mediators by a single molecule, and thus provides an advantage. Anti-Ang2 antibodies negates the destabilisation of endothelium caused by Ang-2 and may normalise the pathological ocular vascularisation, thus a bispecific antibody

$\triangle$ Ashish Sharma

drashish79@hotmail.com

1 Department of Vitreoretina, Lotus Eye Hospital and Institute, Coimbatore, TN, India

2 Gavin Herbert Eye Institute, University of California, Irvine, CA, USA

3 Department of Ophthalmology, University Vita-Salute Scientific Institute San Raffaele, Milano, Italy

4 Division of Ophthalmology, Tel Aviv Sourasky Medical Center and Sackler Faculty of Medicine, Tel Aviv University, Tel Aviv, Israel
(bsAb) with anti-Ang2 and anti-VEGF domain was an ideal candidate to be evaluated.

Faricimab was developed as RG7716 on CrossMAB platform developed by Roche (Basel, Switzerland) to produce next generation biologically engineered bispecific molecule (Fig. 1). CrossMAB is a proprietary technology that ensures heterodimerisation of two different antigen binding domains in a single molecule $[5,6]$. This process utilises knobs and holes format along with a LEGO bricklike structure to avoid the chain association issue and ensures a product with no side products due to linking between undesired chains, known as Bence Jones mAb [7]. Preclinical data have shown that the dual action of RG7716 has shown greater promise to stabilise the vascular leakage from a spontaneous choroidal neovascularisation $(\mathrm{CNV})$ in a mouse model or a LASER-induced CNV in primates compared to the monotherapy of either agent. It also has shown anti-inflammatory properties in cases of endotoxininduced uveitis mouse models [8]. Phase I clinical trial analysed 24 patients of neovascular age-related macular degeneration (nAMD) refractory to 3 or more anti-VEGF injections in last 6 months, demonstrated by leakage on fundus fluorescein angiography (FFA) or presence of fluid on optical coherence tomography (OCT). It exhibited the molecule to have an overall favourable safety profile with evidence of best corrected visual acuity (BCVA) and anatomical improvement [9].

Ang2 upregulation was noted in conditions such as hypoxia, hyperglycaemia and oxidative stress. Blocking Ang2 will stabilise the pericyte loss, and inhibiting Ang2integrin receptor mediated endothelial tip sprouting. Faricimab was hypothesised to be effective in diabetic macular oedema (DME) by invoking this mechanism along with anti-VEGF action. Phase II clinical trials of faricimab were initiated for both DME and nAMD, dubbed as BOULEVARD and STAIRWAY trials, respectively. BOULEVARD was a 36-week, double blind, randomised, active comparator controlled multicentric trial recruiting 229 patients of DME in three treatment cohorts. A total of 168 patients were treatment naïve while 61 patients had received 


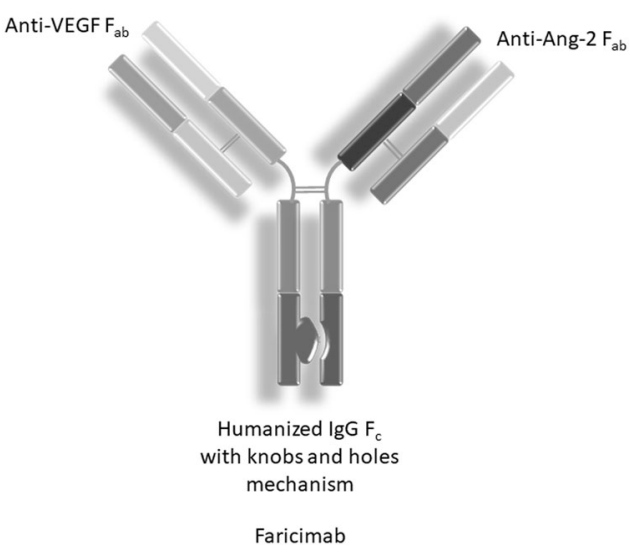

Fig. 1 Faricimab structure

anti-VEGF injections previously [10]. Treatment-naïve patients were randomised into three cohorts who received $6.0 \mathrm{mg}$ of faricimab, $1.5 \mathrm{mg}$ of faricimab and $0.3 \mathrm{mg}$ of ranibizumab. Previously treated patients were randomised into $6.0 \mathrm{mg}$ of faricimab and $0.3 \mathrm{mg}$ of ranibizumab. A total of $6.0 \mathrm{mg}$ was the maximum feasible dose in $50 \mu \mathrm{l}$ of faricimab, which has four times molar concentration than $0.5 \mathrm{mg}$ of ranibizumab while $1.5 \mathrm{mg}$ of faricimab has the similar molar anti-VEGF dose. $1.5 \mathrm{mg}$ arm of faricimab thus allowed for evaluation of an additional Ang-2 inhibition with comparable anti-VEGF dosing to ranibizumab arm. The participants were administered the drug in masked fashion every 4 weeks (q4w) for 20 weeks and primary endpoint outcomes were measured at 24 weeks, and were followed up till 36 weeks. The trial showed superiority of $6.0 \mathrm{mg}$ faricimab arm over $0.3 \mathrm{mg}$ ranibizumab arm in terms of letter gains in visual acuity, greater central subfield thickness (CST) reduction and diabetic retinopathy severity score (DRSS) improvement. It also has shown to have better durability in improvement when compared to ranibizumab, i.e. the patients treated with faricimab had less requirement of repeat injection in follow-up period. This has a potential to establish treat and extend (TREX) regimen in DME.

STAIRWAY trial evaluated two extended dosing regimens of faricimab in 76 patients of nAMD. A total of $6.0 \mathrm{mg}$ of faricimab was given in four weekly loading doses followed by two different dosing schedules of q16w and $\mathrm{q} 12 \mathrm{w}$ dosing and compared to $0.5 \mathrm{mg}$ of ranibizumab in $\mathrm{q} 4 \mathrm{w}$ dosing. The unpublished data have revealed that faricimab has a potential of q16w dosing in nearly two-thirds of the patients. Faricimab and ranibizumab had comparable reduction of central subfoveal thickness (CST) in both dosing regimens [11]. Both phase II trials of faricimab have shown to be safe and effective option for treating DME and nAMD. The molecule has now progressed to RHINE and YOSEMITE phase III trials for DME, while its effect on nAMD will be evaluated in TENAYA and LUCERNE studies [12-15]. Phase III will also evaluate the additional anti-inflammatory properties of the drug.

Aflibercept was the first molecule proven to be effective in combating nAMD and DME by trapping VEGF via VEGF receptors attached to a humanised IgG Fc region. Aflibercept has an additional action on placental growth factor (PGF), which has also been implicated in retinal neovascular pathologies $[1,16]$. There have been previous attempts to simultaneously counteract Ang2 and VEGF in the trials with a co-formulated intravitreal injection of nevascumab (a novel Ang2 inhibitor) and aflibercept. The phase II RUBY trial showed no additional benefit of the combination over aflibercept monotherapy in terms of visual gains [17]. This led to cessation of further development of the co-formulation. The molecular linkage between two domains in the bsAb in faricimab has given it the desired superiority and thus may be categorised as 'Obligate bsAb', where the physical linkage gives an additional therapeutic advantage over the monotherapies [18].

\section{Compliance with ethical standards}

Conflict of interest The authors declare that they have no conflict of interest.

Publisher's note Springer Nature remains neutral with regard to jurisdictional claims in published maps and institutional affiliations.

\section{References}

1. Campochiaro PA. Molecular pathogenesis of retinal and choroidal vascular diseases. Prog Retin Eye Res. 2015;49:67-81.

2. Maisonpierre PC, Suri C, Jones PF, Bartunkova S, Wiegand SJ, Radziejewski C, et al. Angiopoietin-2, a natural antagonist for Tie2 that disrupts in vivo angiogenesis. Science. 1997;277:55-60.

3. Yancopoulos GD, Davis S, Gale NW, Rudge JS, Wiegand SJ, Holash J. Vascular-specific growth factors and blood vessel formation. Nature. 2000;407:242-8.

4. Benest AV, Kruse K, Savant S, Thomas M, Laib AM, Loos EK, et al. Angiopoietin-2 is critical for cytokine-induced vascular leakage. PLoS ONE. 2013;8:e70459.

5. Schaefer W, Regula JT, Bahner M, Schanzer J, Croasdale R, Durr $\mathrm{H}$, et al. Immunoglobulin domain crossover as a generic approach for the production of bispecific IgG antibodies. Proc Natl Acad Sci USA. 2011;108:11187-92.

6. Regula JT, Lundh von Leithner P, Foxton R, Barathi VA, Cheung CMG, Bo Tun SB, et al. Targeting key angiogenic pathways with a bispecific CrossMAb optimized for neovascular eye diseases. EMBO Mol Med. 2016;8:1265-88.

7. Klein C, Schaefer W, Regula JT, Dumontet C, Brinkmann U, Bacac M, et al. Engineering therapeutic bispecific antibodies using CrossMab technology. Methods. 2019;154:21-31.

8. Iwata D, von Leithner PL, Ng YSE, Hartmann G, Shima DT. AntiVEGF/Ang2 bi-specific antibody ameliorates endotoxin-induced uveitis in mice. Invest Ophthalmol Vis Sci. 2014;55:2354.

9. Chakravarthy U, Bailey C, Brown D, Campochiaro P, Chittum M, Csaky K, et al. Phase I trial of anti-vascular endothelial growth factor/anti-angiopoietin 2 bispecific antibody RG7716 for 
neovascular age-related macular degeneration. Ophthalmol Retina. 2017;1:474-85.

10. Sahni J, Patel SS, Dugel PU, Khanani AM, Jhaveri CD, Wykoff $\mathrm{CC}$, et al. Simultaneous inhibition of angiopoietin-2 and vascular endothelial growth factor-A with faricimab in diabetic macular edema. Ophthalmology. 2019;126:1155-1170.

11. Khanani AM, et al. Simultaneous inhibition of VEGF and Ang-2 with faricimab in neovascular AMD: STAIRWAY phase 2 results. Presented at the 2018 American Academy of Ophthalmology (AAO) Annual Meeting; 26 Oct 2018; Chicago, United States.

12. ClinicalTrials.gov. A study to evaluate the efficacy and safety of faricimab (RO6867461) in participants with diabetic macular edema. https://clinicaltrials.gov/ct2/show/NCT03622593. Accessed $15 \mathrm{Jul}$ 2019.

13. ClinicalTrials.gov. A study to evaluate the efficacy and safety of faricimab (RO6867461) in participants with diabetic macular edema (YOSEMITE). https://clinicaltrials.gov/ct2/show/NCT03622580. Accessed 15 Jul 2019.
14. ClinicalTrials.gov. A study to evaluate the efficacy and safety of faricimab in participants with neovascular age-related macular degeneration (TENAYA). https://clinicaltrials.gov/ct2/show/ NCT03823287. Accessed 15 Jul 2019.

15. ClinicalTrials.gov. A study to evaluate the efficacy and safety of faricimab in participants with neovascular age-related macular degeneration (LUCERNE). https://clinicaltrials.gov/ct2/show/ NCT03823300. Accessed 15 Jul 2019.

16. Papadopoulos N, Martin J, Ruan Q, Rafique A, Rosconi MP, Shi E, et al. Binding and neutralization of vascular endothelial growth factor (VEGF) and related ligands by VEGF Trap, ranibizumab and bevacizumab. Angiogenesis. 2012;15:171-85.

17. Boyer DS. Intravitreal nesvacumab+aflibercept in diabetic macular edema: The Phase 2 RUBY Trial. Invest. Ophthalmol. Vis. Sci. 2018;59:3620.

18. Labrijn AF, Janmaat ML, Reichert JM, Parren PWHI. Bispecific antibodies: a mechanistic review of the pipeline. Nat Rev Drug Discov. 2019;18:585-608. 